\title{
AVALIAÇÃO DO USO DO DODECANOL NA FERMENTAÇÃO EXTRATIVA LÍQUIDO-LÍQUIDO ATRAVÉS DE UMA PLATAFORMA VIRTUAL
}

\author{
G . P. ALMEIDA ${ }^{1}$, G. H. S. F. PONCE ${ }^{1}$, M. R. W. MACIEL ${ }^{1}$ e R. M. FILHO ${ }^{1}$ \\ ${ }^{1}$ Universidade de Campinas, Faculdade de Engenharia Química, Departamento de \\ Desenvolvimento de Processos e Produtos \\ E-mail para contato: guilherme@ feq.unicamp.br
}

\begin{abstract}
RESUMO - As etapas de separação nos processos de produção de etanol de primeira e segunda gerações são de grande interesse na indústria química. A técnica de extração líquido-líquido (ELL) com solventes biocompatíveis tem se apresentado como uma tecnologia em potencial na extração do etanol já que não requer altas temperaturas e é aplicada para soluções diluídas. Nesse contexto, a ELL acoplada ao fermentador foi estudada utilizando o simulador ASPEN PLUS®, para diferentes vazões do solvente dodecanol e temperaturas $\left(25^{\circ} \mathrm{C}\right.$ a $\left.35^{\circ} \mathrm{C}\right)$. Os diagramas ternários simulados com o modelo NRTL foram validados com dados experimentais da literatura. Dentre os solventes analisados, o dodecanol, em baixas temperaturas, conseguiu extrair todo o etanol produzido na fermentação para uma razão solvente/alimentação de aproximadamente 5,0. A temperatura não interfere significativamente na ELL do etanol. Os resultados apresentados neste trabalho comprovaram a viabilidade do uso de solventes biocompatíveis para recuperação do etanol.
\end{abstract}

\section{INTRODUÇÃO}

As mudanças globais em termos de tecnologia, meio ambiente e condições econômicas fizeram com que o etanol de $1^{\text {a }}$ geração, deixasse de ser uma possibilidade energética para se tornar uma fonte renovável viável e competitiva frente ao petróleo. O processo de produção do etanol de $1^{\text {a }}$ geração consiste basicamente na extração do caldo de cana-de-açúcar, em seguida, ocorre a etapa de fermentação pela ação de uma levedura, geralmente Saccharomyces cerevisiae, e em sequencia a separação do etanol (Cardona et al., 2010).

Apesar da consolidação do processo de produção do etanol de $1^{\mathrm{a}}$ geração, a etapa de fermentação e recuperação do etanol ainda é fonte de inúmeras pesquisas. Dentre os gargalos do processo, está a inibição da fermentação pelo próprio etanol às baixas concentrações, e consequentemente, a necessidade da remoção do etanol do meio fermentativo, o que requer grande quantidade de energia. Segundo Yokoya et al. (1989), concentrações de etanol no mosto em torno de $10 \% \mathrm{~V} / \mathrm{V}$ já tornam o meio desfavorável para o crescimento da levedura. Minier \& Goma (1982) observaram que o crescimento celular cessa ao se atingir uma concentração de etanol do mosto de $12^{\circ} \mathrm{GL}$, o que equivale aproximadamente a $95 \mathrm{~g} / \mathrm{L}$. Para contornar esse problema da inibição da levedura pelo etanol, operações de remoção contínua do etanol acopladas ao fermentador (extração in situ) têm sido estudadas, dentre elas a extração líquidolíquido (ELL) com solventes biocompatíveis. 
A técnica de ELL, utilizando o solvente e o projeto adequado é eficiente, econômica e não poluente. Além disso, a inibição da levedura pela baixa concentração de etanol coloca a técnica de ELL como uma possível alternativa de separação já que essa operação é aplicada para misturas diluídas. Porém, para que a ELL possa ser acoplada ao fermentador, é necessário que o solvente não iniba o crescimento da levedura. Na literatura, os estudos experimentais de Daugulis (1987), Minier e Goma (1982), e Jassal et al. (1994), apresentaram alguns solventes biocompatíveis, óleo oléico, dodecanol e ácido oléico respectivamente. Dentre esses solventes o dodecanol é o que apresenta maior coeficiente de partição, além disso, há uma maior quantidade de dados experimentais na literatura que possam validar a simulação.

A partir da seleção do solvente (dodecanol), o objetivo do trabalho é desenvolver uma plataforma virtual para a ELL acoplada ao fermentador, processo que é denominado fermentação extrativa líquido-líquido. Dessa forma pretende-se simular a extração com solvente in situ para o processo de etanol de $1^{\text {a }}$ geração e investigar as principais variáveis do o processo, a saber: vazão de solvente e temperatura.

\section{SIMULAÇÃO DE EXTRAÇÃO LÍQUIDO-LIQUIDO ACOPLADA AO FERMENTADOR}

O desenvolvimento da plataforma virtual da ELL acoplada ao fermentador foi realizado através do simulador ASPEN PLUS ®V.7.3 (Aspen Plus User Guide, 2011). A matéria-prima utilizada na produção de etanol de $1^{a}$ geração é o caldo da cana de açúcar tratado que é constituído basicamente de água $(75 \% \mathrm{~m} / \mathrm{m})$, fibras e resíduos sólidos $(10 \% \mathrm{~m} / \mathrm{m})$ e sacarose, que pode variar de 10 a $15 \% \mathrm{~m} / \mathrm{m}$ dependendo da variedade da cana. Entretanto, nas fábricas de etanol é comum na produção de etanol se utilizar como fonte de sacarose não apenas o caldo de cana tratado, mas uma mistura de caldo, melaço e caldo de cana evaporado que são provenientes das correntes de processo da fabricação de açúcar (Copersucar, 1987). Em termo de simulação, a corrente de alimentação do fermentador foi aproximada para uma mistura de glicose e água, simplificando as reações químicas no reator, já que no processo real a sacarose contida na mistura é convertida em glicose pela levedura, com uma conversão de 99\% (Dias, 2008). O fermentador foi simulado a partir de um reator RSTOIC@ que é um reator contínuo baseado na estequiometria da reação, modelado a partir das conversões das reações específicas determinadas experimentalmente para levedura Saccharomyces cerevisiae com base no rendimento industrial das indústrias sucroalcooleiras brasileiras. A Tabela 1 apresenta os produtos, as equações estequiométricas e as respectivas conversões a partir da glicose, de acordo com Dias (2008).

Tabela 1: Conversão e equações para formação dos produtos a partir da glicose (Dias, 2008)

\begin{tabular}{|c|c|c|}
\hline Produto & Reação & $\begin{array}{c}\text { Conversão } \\
(\%)\end{array}$ \\
\hline Etanol & $\mathrm{C}_{6} \mathrm{H}_{12} \mathrm{O}_{6} \rightarrow 2 \mathrm{C}_{2} \mathrm{H}_{5} \mathrm{OH}+2 \mathrm{CO}_{2}$ & 90,48 \\
\hline Glicerol & $\mathrm{C}_{6} \mathrm{H}_{12} \mathrm{O}_{6}+4 \mathrm{H}^{+} \rightarrow 2 \mathrm{C}_{3} \mathrm{H}_{8} \mathrm{O}_{3}$ & 2,67 \\
\hline $\begin{array}{c}\text { Ácido } \\
\text { Succínico }\end{array}$ & $\mathrm{C}_{6} \mathrm{H}_{12} \mathrm{O}_{6}+2 \mathrm{H}_{2} \mathrm{O} \rightarrow 2 \mathrm{C}_{4} \mathrm{H}_{6} \mathrm{O}_{4}+2 \mathrm{CO}_{2}+10 \mathrm{H}^{+}+10 \mathrm{e}^{-}$ & 0,29 \\
\hline $\begin{array}{c}\text { Ácido } \\
\text { Acético }\end{array}$ & $\mathrm{C}_{6} \mathrm{H}_{12} \mathrm{O}_{6}+2 \mathrm{H}_{2} \mathrm{O} \rightarrow 2 \mathrm{C}_{4} \mathrm{H}_{4} \mathrm{O}_{2}+2 \mathrm{CO}_{2}+8 \mathrm{H}^{+}+8 \mathrm{e}^{-}$ & 1,19 \\
\hline $\begin{array}{c}\text { Ácido } \\
\text { Isoamílico }\end{array}$ & $\mathrm{C}_{6} \mathrm{H}_{12} \mathrm{O}_{6} \rightarrow 0,795 \mathrm{C}_{5} \mathrm{H}_{12} \mathrm{O}+1,155 \mathrm{H}_{2} \mathrm{O}+2,025 \mathrm{CO}_{2}+0,15 \mathrm{H}^{+}+0,15 \mathrm{e}^{-}$ & $3,1.10^{-4}$ \\
\hline
\end{tabular}


Levedura

(Zymo)

$$
\mathrm{C}_{6} \mathrm{H}_{12} \mathrm{O}_{6}+1,143 \mathrm{NH}_{3} \rightarrow 5,7143 \mathrm{Zymo}+2,57 \mathrm{H}_{2} \mathrm{O}+0,2857 \mathrm{CO}_{2}
$$

A Saccharomyces cerevisiae é a levedura que cresce ao longo da fermentação e atua na reação de fermentação alcoólica como catalisador, no entanto, as propriedades desse microrganismo sólido não estão presentes na biblioteca de dados do simulador ASPEN PLUS®. Dessa forma, para estudo de separação e reciclo de células, foi necessário criar a etapa de formação e crescimento da levedura através dos dados do componente denominado Zymo e proposto por Wooley e Putsche (1996), usando os dados fornecidos no Development of an ASPEN PLUS Physical Property Database for Biofuels Components, para o componente Zymo conforme apresentado na Tabela 2.

Tabela 2: Propriedades da Levedura Saccharomyces Cerevisiae e do componente Zymo

\begin{tabular}{|l|c|c|}
\hline \multicolumn{1}{|c|}{ Propriedades } & Levedura & Zymo \\
\hline Capacidade calorífica & - & 0,45 \\
\hline Densidade & 62,43 & 80,00 \\
\hline Fórmula molecular & $\mathrm{CH}_{1,8} \mathrm{O}_{0,9} \mathrm{~N}_{0,145}$ & $\mathrm{CH}_{1,8} \mathrm{O}_{0,5} \mathrm{~N}_{0,20}$ \\
\hline Massa molar & 30,23 & 25,2 \\
\hline
\end{tabular}

O fluxograma do processo fermentativo extrativo é apresentado na Figura 1, o extrator líquidolíquido (EXTRAT) é acoplado ao reator de fermentação contínua, fazendo com que o etanol seja retirado ao mesmo tempo em que é produzido, permitindo que sua concentração no reator permaneça em níveis baixos ao longo do processo. Dessa maneira, a inibição da levedura pelo produto diminui, aumentando a produtividade do processo. O reator opera de forma semelhante a um reator contínuo convencional: o mosto e as células de levedura (pé de cuba) são adicionados de forma contínua ao reator e o vinho levedurado (vinho contendo leveduras) é retirado também de forma contínua (VINHO). O vinho levedurado obtido no reator de fermentação passa por um separador flash que separa o gás carbônico formado principalmente na reação de conversão da glicose em etanol. Em seguida o vinho (VINHOLEV) contendo água, açúcar não convertidos, etanol, levedura, glicerol e outros ácidos como o ácido acético são enviados para coluna extratora, simulando o processo da extração líquido-líquido do etanol in situ. A vazão de solvente tem entrada na parte inferior da coluna extratora tendo em vista que a densidade do dodecanol $(\rho=0,83 \mathrm{~g} / \mathrm{mL})$ é menor que a densidade do vinho delevedurado $(\rho \sim 1,00 \mathrm{~g} / \mathrm{mL})$, uma vez que este contém aproximadamente $90 \%$ de água em massa. O produto de topo (EXTRATO) contém principalmente etanol, água e o dodecanol que seguirá para a recuperação do etanol hidratado ou anidro, etapa esta não avaliada no presente trabalho. A corrente de fundo (RAF) do extrator contém água, o excesso de nutrientes, ácidos carboxílicos, glicerol, açúcares não convertidos, biomassa, levedura e etanol que segue para o processo de remoção e reciclo das células de levedura. $\mathrm{O}$ processo de separação consiste na passagem do vinho levedurado por uma centrífuga (CENTRIF), na qual o leite de levedura é separado do rafinado (RAFINADO) que poderá ir para recuperação da água. O fluxo de biomassa é reciclado (RECICL) para o fermentador e o excedente de biomassa é extraído do processo (EXCES). É importante ressaltar que o conjunto REATOR, FLASH e EXTRATOR operam nas mesmas condições de temperatura e pressão e representam a ELL in situ que pode ser utilizada como etapa à montante ao destilador concentrando o vinho ou até mesmo em substituição ao processo tradicional de destilação em que o vinho é diretamente enviado para as colunas de destilação, onde é purificado, concentrado e recuperado como etanol hidratado. 


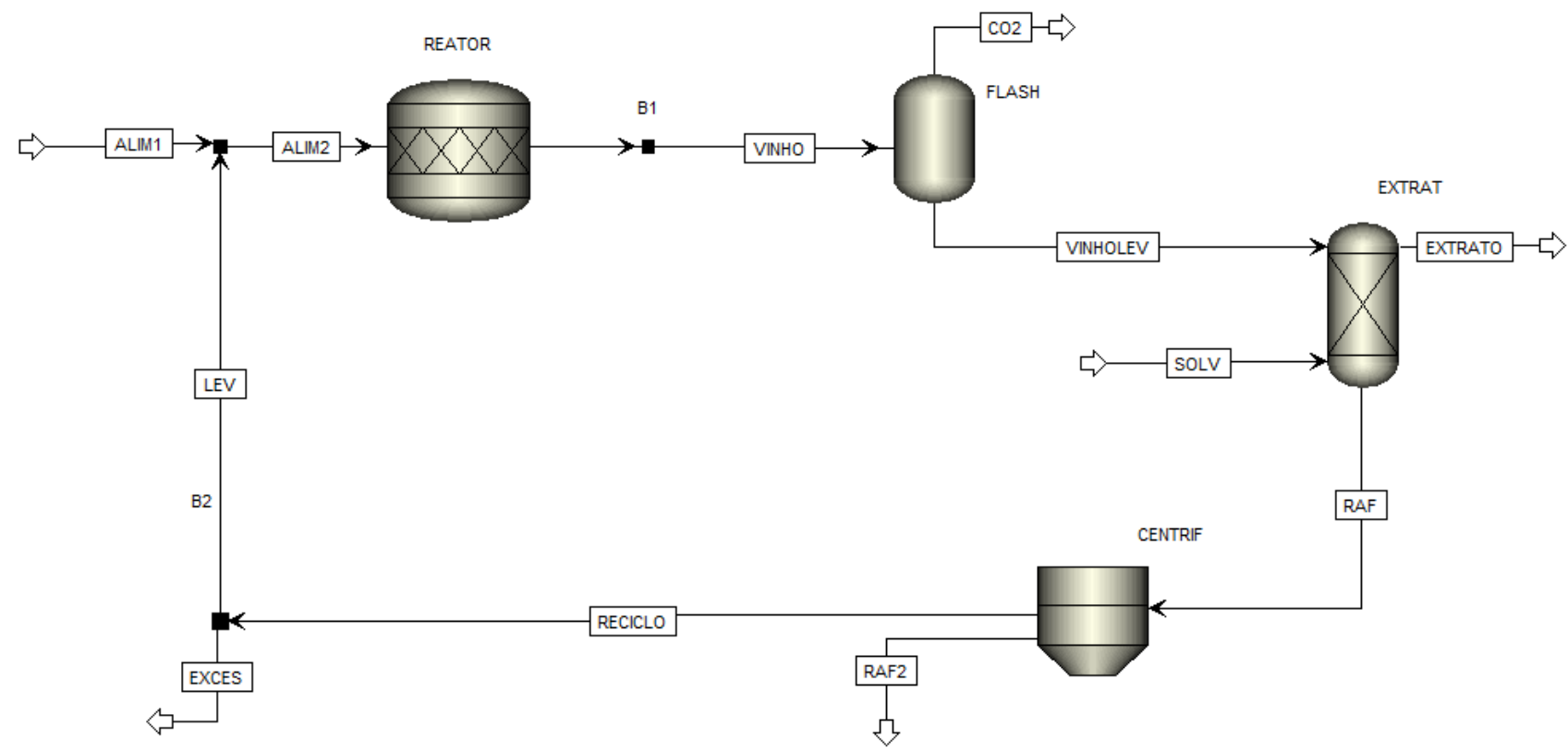

Figura 1 - Fluxograma do processo de fermentação contínua acoplado ao extrator líquido-líquido

O arranjo descrito na Figura 1 acima foi feito com base nos trabalhos de Van Der Merwe (2010) e Ponce et al. (2014) que estudaram a remoção do etanol in situ através de gás stripping. No presente trabalho, o separador flash e o extrator são simulados com as mesmas condições de temperatura e pressão do fermentador. O modelo Non-Random Two Liquid (NRTL) foi escolhido por seu bom desempenho para representar o equilíbrio líquido-líquido de misturas altamente não ideais.

\section{RESULTADOS E DISCUSSÃO}

Primeiramente, com o objetivo de verificar a precisão de estimativa dos dados de equilíbrio utilizando o modelo NRTL, foram construídos diagramas ternários, indicados nas Figuras 2 e 3 utilizando-se o simulador ASPEN PLUS ${ }^{\circledR}$ para as temperaturas de $25^{\circ} \mathrm{C}$ e $35^{\circ} \mathrm{C}$, respectivamente. Nos mesmos gráficos, foram plotados os resultados experimentais em vermelho do trabalho de Kirbaslar et al. (2001). A região pontilhada, abaixo da curva binodal, representa a mistura heterogênea do sistema em que há formação de duas fases, uma rica em água e outra rica em solvente (dodecanol). A região acima da curva indica uma mistura homogênea. Por meio da Figura 2, é possível concluir que o modelo escolhido NRTL descreve bem os dados experimentais para o sistema etanol-água-dodecanol a $25^{\circ} \mathrm{C}$, com desvios em torno de $3 \%$. 


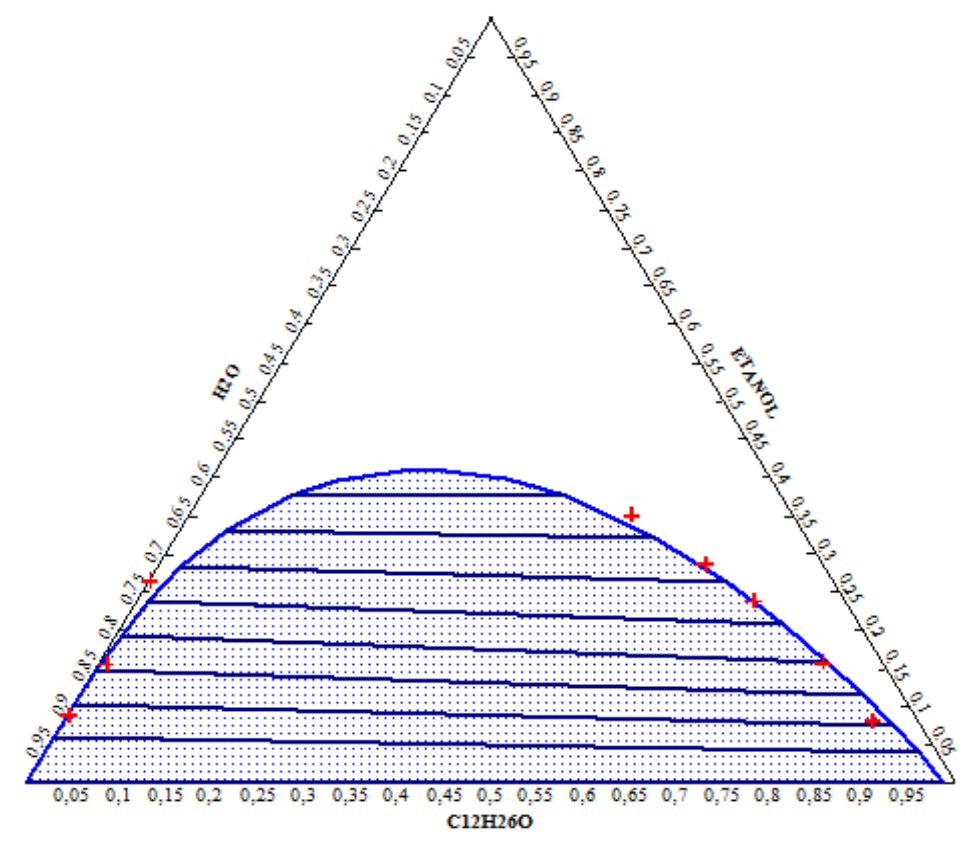

Figura 2 - Diagrama de equilíbrio líquido-líquido para Água-Dodecanol $\left(\mathrm{C}_{12} \mathrm{H}_{26} \mathrm{O}\right)$-Etanol a $25^{\circ} \mathrm{C}$ sendo $(-)$ para o modelo NRTL e $(+)$ para os dados experimentais de Kirbaslar et al (2001).

Já para o sistema a $35^{\circ} \mathrm{C}$ apresentado na Figura 3, têm-se desvios maiores para o modelo NRTL (em torno de 6\%). Além disso, ao comparar os sistemas nas duas temperaturas, observase que a variação de temperatura (na faixa de temperaturas estudadas) não interferiu de forma significativa na curva binodal.

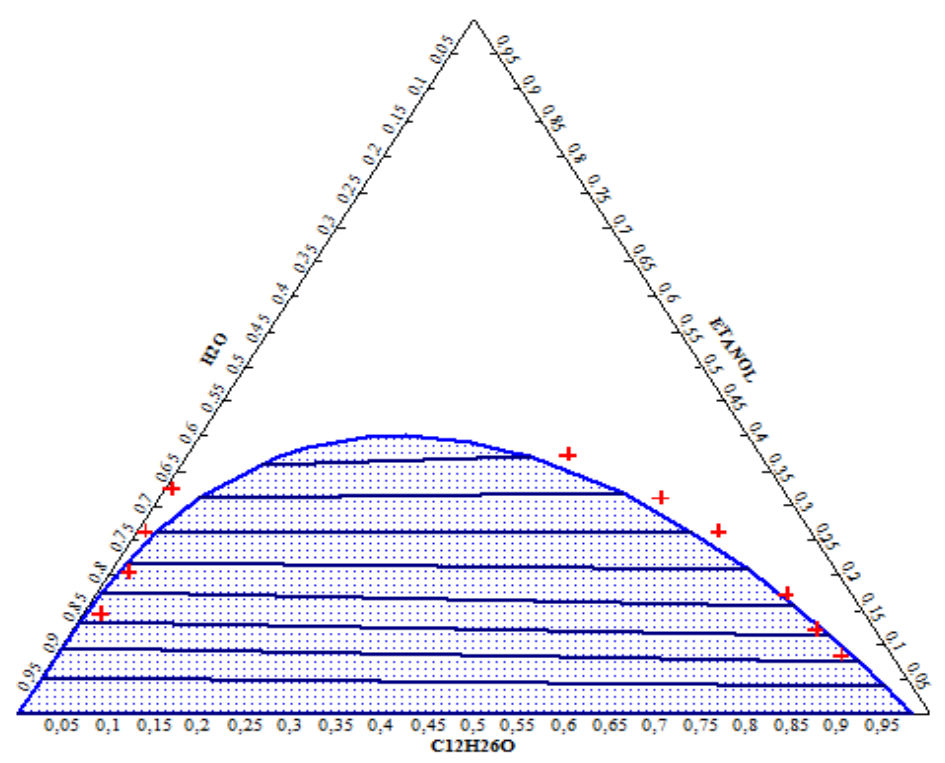

Figura 3 - Diagrama de equilíbrio líquido-líquido para Água-Dodecanol $\left(\mathrm{C}_{12} \mathrm{H}_{26} \mathrm{O}\right)$-Etanol a $35^{\circ} \mathrm{C}$ sendo $(-)$ para o modelo NRTL e $(+)$ para os dados experimentais de Kirbaslar et al (2001). 
A partir da validação do modelo termodinâmico NRTL a fermentação extrativa líquidolíquido foi simulada a 1 atm, utilizando um extrator com 2 estágios de forma que representasse uma única operação in situ fermentador, flash e extrator. No processo de produção e extração do etanol estudado, a fermentação ocorre de forma contínua a $30^{\circ} \mathrm{C}$, com uma corrente de $50 \mathrm{Kg} / \mathrm{h}$ de solvente para uma entrada de $100 \mathrm{Kg} / \mathrm{h}$ de Alimentação (ALIM1) do fermentador que contém $18 \% \mathrm{~m} / \mathrm{m}$ de glicose. A fração de glicose utilizada na simulação representa valores próximos aos utilizados no processo industrial do etanol de $1^{\text {a }}$ geração. As respostas das principais correntes e componentes para o processo estudado são apresentadas na Tabela 3.

Tabela 3 - Dados das correntes para fluxograma do processo da Figura 1

\begin{tabular}{|c|c|c|c|c|c|c|c|}
\hline & VINHO & $\mathrm{CO}_{2}$ & VINHOLEV & EXTRATO & RAF & RAF2 & RECICL \\
\hline $\begin{array}{c}\text { Vazão Mássica } \\
(\mathrm{Kg} / \mathrm{h})\end{array}$ & 101,7917 & 8,1218 & 93,6697 & 54,9617 & 88,702 & 86,5637 & 2,10 \\
\hline Temperatura $\left({ }^{\circ} \mathrm{C}\right)$ & 29,0 & 30,0 & 30,0 & 28,35 & 26,19 & 26,19 & 26,59 \\
\hline Glicose $(\mathrm{m} / \mathrm{m})$ & 0,0072 & 0,0000 & 0,0078 & 0,0016 & 0,0072 & 0,0072 & 0,0068 \\
\hline Água $(\mathrm{m} / \mathrm{m})$ & 0,8223 & 0,0169 & 0,8935 & 0,0286 & 0,9265 & 0,9265 & 0,1150 \\
\hline Etanol $(\mathrm{m} / \mathrm{m})$ & 0,0830 & 0,0160 & 0,0889 & 0,0573 & 0,0577 & 0,0577 & 0,0071 \\
\hline $\mathrm{CO}_{2}(\mathrm{~m} / \mathrm{m})$ & 0,0800 & 0,9664 & 0,0016 & 0,0025 & 0,0001 & 0,0001 & 0,0073 \\
\hline Glicerol $(\mathrm{m} / \mathrm{m})$ & 0,0049 & 0,0000 & 0,0053 & 0,0000 & 0,0056 & 0,0056 & 0,0007 \\
\hline Acid. Acét. $(\mathrm{m} / \mathrm{m})$ & 0,0014 & 0,0000 & 0,0015 & 0,0003 & 0,0014 & 0,0014 & 0,0000 \\
\hline Dodecanol $(\mathrm{m} / \mathrm{m})$ & 0,0000 & 0,0000 & 0,0000 & 0,9093 & 0,0002 & 0,0002 & 0,0001 \\
\hline Levedura $(\mathrm{m} / \mathrm{m})$ & 0,01768 & 0,0000 & 0,017768 & 0,0000 & 0,0213 & 0,0000 & 0,8795 \\
\hline
\end{tabular}

Os resultados da Tabela 3 mostram uma concentração 8,30\% $\mathrm{m} / \mathrm{m}$ de etanol na corrente de saída do fermentador (VINHO). Essa concentração diminui para $5,77 \% \mathrm{~m} / \mathrm{m}$ na corrente de rafinado após a extração com o dodecanol. Além disso, os subprodutos, como o glicerol, ácido succínico e ácido acético e a levedura foram concentrados na corrente de rafinado (RAF). Ao se avaliar a eficiência da extração líquido-líquido nas condições estudadas, tem-se na corrente de extrato (EXTRATO) todo o solvente, o dodecanol e, aproximadamente, 38,6\% do etanol produzido no fermentador é recuperado o que facilitaria a etapa de destilação para obtenção do etanol hidratado ou puro (método tradicional). Nas saídas do vaso Flash tem se a maior parte (98\%) do gás $\mathrm{CO}_{2}$ formado na fermentação na corrente de $\mathrm{CO}_{2}$ que arrasta parte do etanol, gerando uma perda de $2 \%$ de massa de etanol, portanto é interessante utilizar um absorvedor na corrente de $\mathrm{CO}_{2}$ para que o etanol possa ser enviado ao extrator líquido-líquido.

Os efeitos da razão de solvente/alimentação e temperatura na extração do etanol foram avaliados através de análises de sensibilidade. A Figura 4 apresenta a eficiência da separação em função da razão de solvente/alimentação (S/A) do reator a uma temperatura de $30^{\circ} \mathrm{C}$. A percentagem de etanol recuperado do caldo fermentado aumenta com a razão S/A até atingir o ponto máximo em que a eficiência é 1; nessa situação tem-se uma razão solvente/alimentação de aproximadamente 5,0 o que implica em grande quantidade de solvente e, ao mesmo tempo, equipamentos de maior volume o que pode encarecer os custos de projeto da planta. Além disso, à medida que o etanol é concentrado na corrente extrato, maior quantidade de água é arrastada, o que pode prejudicar a eficiência nas futuras etapas de separação e obtenção do etanol anidro ou hidratado. Apesar de todos os problemas que uma vazão de solvente alta implica, ressalta-se que o solvente dodecanol pode ser reutilizado no extrator após a recuperação. 


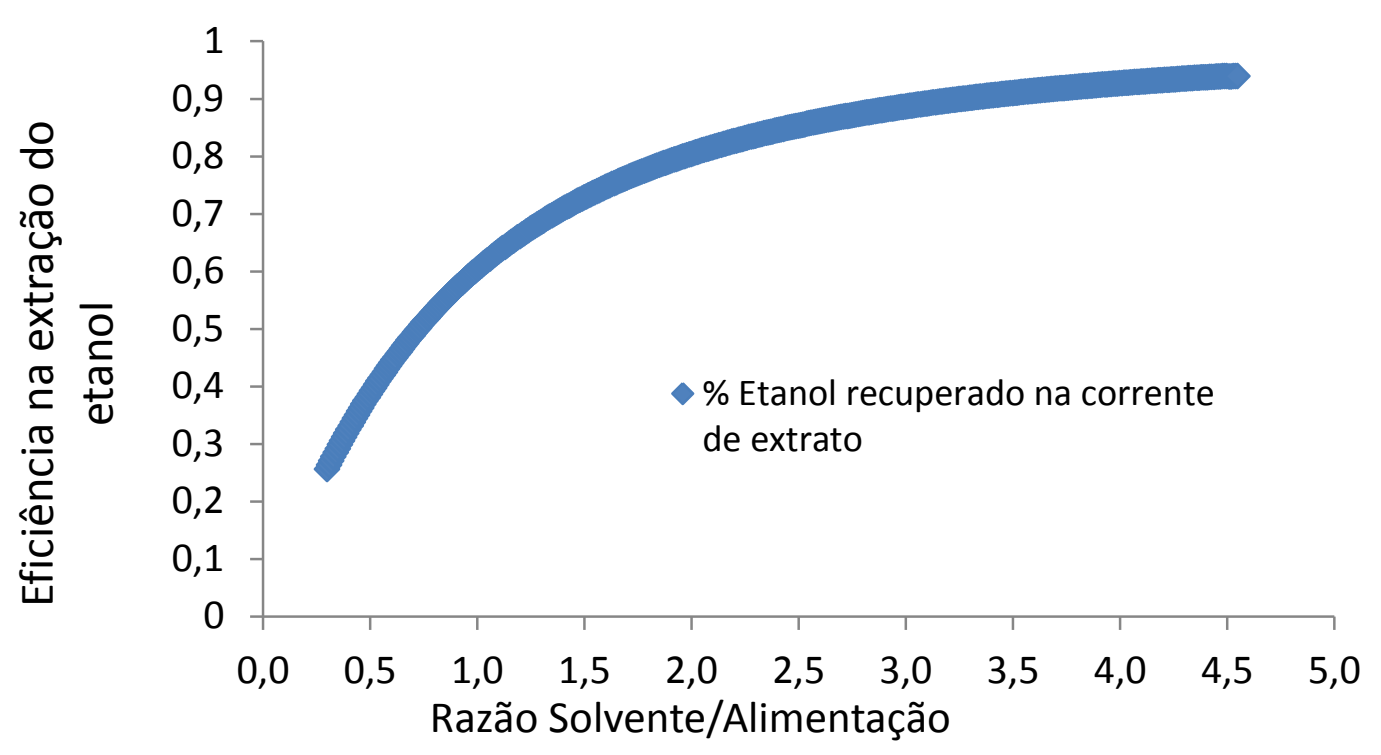

Figura 4 - Gráfico da porcentagem de etanol recuperado do fermentador em função da razão de solvente/alimentação a $30^{\circ} \mathrm{C}$

Uma análise de sensibilidade foi realizada variando-se a temperatura do reator de $25^{\circ} \mathrm{C}$ a $35^{\circ} \mathrm{C}$, porém não foi observada variação significativa para a extração do etanol, o que é confirmado pelos diagramas ternários apresentados anteriormente (Figuras 2 e 3 ) onde não foi observado variação significativa na curva binodal. Como no caso de estudo a levedura está em contato com o solvente no extrator, é importante ressaltar que temperaturas acima de $35^{\circ} \mathrm{C}$ afetam o crescimento e comportamento da levedura, diminuindo a conversão da glicose em etanol, dessa maneira temperaturas acima de $35^{\circ} \mathrm{C}$ não foram estudadas (Paternina, 2011).

\section{CONCLUSÃO}

A plataforma virtual que descreve o processo de extração líquido-líquido acoplada ao fermentador foi avaliada através de resultados experimentais encontrados na literatura para o equilíbrio líquido-líquido do sistema etanol, água e dodecanol. O modelo NRTL se mostrou preciso para os dados experimentais encontrados confirmando a baixa influência da temperatura no ELL com o dodecanol. Entretanto, como o caso é de uma extração in situ, a temperatura torna-se um fator importante na fermentação tendo em vista a alta sensibilidade do crescimento da levedura com a temperatura, que é interrompido a $35^{\circ} \mathrm{C}$. A eficiência da separação do etanol para a corrente de topo (extrato) apresenta seu máximo quando a vazão S/A (solvente/alimentação) atinge uma razão de 5,0 e atinge uma eficiência de aproximadamente 40 \% para uma razão de $0,5 \mathrm{~S} / \mathrm{A}$. Nessa razão $(0,5)$ uma menor quantidade de dodecanol é consumida e quantidades significativas de etanol podem ser concentradas nas correntes destinadas às colunas de destilação; assim, a ELL in situ poderia ser utilizada como complemento ao processo tradicional. Além dessa vantagem, a ELL acoplada ao fermentador ocorre na temperatura da fermentação, sem a necessidade de aquecimento. A plataforma virtual para a fermentação extrativa líquido-líquido será utilizada para futuros estudos de reciclo de solvente e recuperação do etanol nas correntes de extrato, rafinado e vapor, avaliando-se os custos energéticos do processo. 


\section{REFERÊNCIAS}

ASPEN PLUS USER GUIDE, Aspen Technology, Inc. AspenTech Version 7.3. Burlington, USA, 2011.

CARDONA, C.A.; SANCHEZ, O.J.; GUTIERREZ, L.F. Process Synthesis for Fuel Ethanol Production. CRC Press, Boca Raton, 2010.

COPERSUCAR. Apostila do curso de Engenharia açucareira: Processo de Fabricação de Açúcar, parte I. Piracicaba, 1987.

DAUGULIS, A. J.; AXFORD, D. B.; CISZEK, B.; MALINOWSKI, J. J. Continuous fermentation of high-strength glucose feeds to ethanol. Biotechnology and bioengineering, v. 16, n. 6, p. 637-642, 1994.

DIAS M.O. Simulation of ethanol production processes from sugar and sugarcane bagasse, aiming process integration and maximization of energy and bagasse surplus. Campinas: Universidade de Campinas, Brasil, 2008.

JASSAL, D. S.; ZHANG, Z.; HILL, G.A. In-situ Extraction and Purification of Ethanol Using Commercial Oleic Acid. Can. J. Chem. Eng., v. 72, p. 822-826, 1994.

KIRBASLAR, S. I.; ÇEHRELI, D. U.; KESKINOCAK, E. Equilibrium Data on Water-Ethanol1-Dodecanol Ternary System. Turk Journal Engineering Science, v. 25, p. 111-115, 2001.

MINIER, M.; GOMA, G. Ethanol production by extractive fermentation. Biotechnology and bioengineering. New York. v. 24, p. 1565-1579, 1982.

PATERNINA, L. M. C. Modelagem e simulação do processo de fermentação extrativa a vácuo com uma câmara de flash e separação do $\mathrm{CO}_{2}$ utilizando uma coluna de absorção. Campinas: Universidade de Campinas, Brasil, 2011.

PONCE, G. H. S. F.; MIRANDA, J. C. C.; ALVES, M.; WOLF, M. R. M.; FILHO, R. M.; ANDRADE, R. R.; CONTO, L. C. Simulation, Analysis and Optimization of an In Situ Gas Stripping Fermentation Process in a Laboratory Scale for Bioethanol Production. Chemical Engineering Transactions, v. 37, p. 295-300, 2014.

VAN DER MERWE A. B. Evaluation of different process design for biobutanol production from sugarcane molasses, Stellenbosch: University of Stellenbosch, South Africa, 2010.

YOKOYA, F. Pontos críticos microbiológicos em usinas de açúcar e álcool. Microbiologia de processo de fermentação. In: Eguchi, S. Y.; Yokoya, F.; Canhos, V. P.; Gallo, C. R., p. 1112, 1989.

WOOLEY, R. J.; PUTSCHE, V. Development of an Aspen Plus physical property database for biofuels components. National Renewable Energy Labortory, 1996. 